We wanted to evaluate whether videoed presentations would be an acceptable medium for delivering the educational component of an outpatient PR programme.

Method Educational sessions were delivered by the multidisciplinary team at a large teaching hospital, and were professionally filmed. Talks covered were: disease education, healthy eating, medicines, avoidance and exacerbations, exercise and activity, energy conservation, relaxation, managing breathlessness and chest clearance. Patients undertaking PR were asked to evaluate the content and delivery of education sessions using a feedback questionnaire. One patient group evaluated the spoken sessions and a second group evaluated the DVDs. Patients' knowledge was assessed with The Bristol COPD Knowledge Questionnaire (BCKQ) before and after rehabilitation.

Results 117 patients completed feedback forms. A maximum of 69 rated the DVD sessions; mean (SD) age 71.13 (9.69), $\mathrm{FEV}_{1} 1.25$ (0.55) 1 , COPD $79.7 \%$. A maximum of 48 patients rated the spoken sessions; mean (SD) age 64.94 (12.59), FEV 1.54 (0.63) 1, COPD $69.8 \%$. Not all patients rated all sessions. Delivery was rated as satisfactory or better by $99.16 \%$ of patients and $99.49 \%$ of patients for DVD and spoken groups, respectively. Initial mean (SD) BCKO scores were 28.34 (10.30) and 26.41 (13.92) for the DVD and talk group, respectively. Both groups improved $\mathrm{BCKO}$ scores following PR; mean (SD) change 4.53 (10.11) for DVD group and 7.36 (8.98) for talk group, but there were no between group differences $(\mathrm{p}=0.905)$.

Conclusion PR patients perceive education delivered by DVD acceptable both in content and medium of delivery. DVDs may be a feasible alternative if multidisciplinary speakers cannot be arranged. This educational medium may also be helpful in reducing the overall costs of rehabilitation, or, if DVDs were to be watched in patients' homes, increasing programme capacity by reducing the duration of each supervised session. For example; for a rolling programme the cost is approximately $£ 1747.60$ per programme, per speaker (Agenda for Change Band 6).

\section{P145 EFFECT OF PULMONARY REHABILITATION ON WAIST CIRCUMFERENCE AND WAIST-HIP RATIO}

\section{doi:10.1136/thoraxjn-2011-201054c.145}

K A Ingram, R P Fowler, A L Clark, P L Marns, M S Patel, S S C Kon, J L Canavan, W D C Man. Harefield Pulmonary Rehabilitation Team and Respiratory Biomedical Research Unit, Royal Brompton \& Harefield NHS Foundation Trust, Harefield, UK

Background Patients with chronic obstructive pulmonary disease (COPD) have increased cardiovascular risk. In selected COPD patients, recent studies have shown that pulmonary rehabilitation (PR) may improve markers of cardiovascular risk, such as arterial stiffness, blood pressure and fasting cholesterol. Waist circumference and waist-hip ratio are related to increased risk of cardiovascular disease, type II diabetes and mortality. We investigated the effect of PR on waist circumference and waist-hip ratio in an unselected population referred for PR.

Methods 256 consecutive patients (181 COPD; 117F:139M; median age 69; median $\mathrm{FEV}_{1} 49 \%$ predicted) completing an eight week outpatient PR programme were analysed. Waist circumference, waist-hip ratio and body mass index (BMI) were measured immediately before and after PR. Wilcoxon signed rank test was used to test the effect of PR.

Results Following PR, there were significant improvements in incremental shuttle walk (ISW) and chronic respiratory disease questionnaire (CRDQ) score (Abstract P145 table 1). There were very small, but statistically significant, reductions in weight, BMI and waist circumference but no changes in waist-hip ratio.
Abstract P145 Table 1 Effect of pulmonary rehabilitation (PR) on incremental shuttle walk (ISW), chronic respiratory disease questionnaire (CRDO), waist circumference and waist hip ratio

\begin{tabular}{lccr}
\hline Outcome measure & Pre-PR & Post-PR & p Value \\
\hline ISW (metres) & $180(90,300)$ & $240(140,380)$ & $<0.001$ \\
CRD0 Total & $75.7(20.3)$ & $92.5(75,111)$ & $<0.001$ \\
Weight $(\mathrm{kg})$ & $74(62,88)$ & $73(62,89)$ & 0.004 \\
BMI $\left(\mathrm{kg} / \mathrm{m}^{2}\right)$ & $27.0(23.3,31.8)$ & $26.8(23.1,31.5)$ & 0.002 \\
Waist circumference $(\mathrm{cm})$ & $100(90,111)$ & $98(89,111)$ & 0.002 \\
Waist-hip ratio & $0.96(0.89,1.02)$ & $0.96(0.90,1.02)$ & 0.55 \\
\hline
\end{tabular}

Data expressed as median (25th, 75th centiles) or mean (SD).

Conclusions An 8-week outpatient PR programme has no clinically meaningful effect upon waist circumference or waist-hip ratio.

\section{P146 VALIDATION OF THE COPD ASSESSMENT TEST (CAT) IN PULMONARY REHABILITATION: APPLICATION TO A COHORT OF MIXED PULMONARY DISEASES}

doi:10.1136/thoraxjnl-2011-201054c.146

P Lachapelle, M Brosseau, M Houle-Péloquin, C Poirier. Centre Hospitalier de I'Université de Montréal, Montreal, Canada

Introduction The CAT is a new self administered questionnaire developed and validated for the assessment and monitoring of COPD patients. ${ }^{1}$ Recently, it has been showed to be sensitive to pulmonary rehabilitation (PR) in a prospective multicentric COPD study. ${ }^{2}$ The CAT has never been validated in a global PR programme with mixed pulmonary diseases.

Methods We performed a retrospective study of 30 patients who completed the CAT before and after PR in 2010-2011. The cohort included seventeen patients diagnosed with COPD, seven with asthma and six with fibrosis. All participants were referred for an 8 weeks, three times weekly rehabilitation programme. Our primary objective was to validate the CAT in the cohort. We also evaluated six other outcome measures of PR as a secondary endpoint (Abstract P146 table 1). Pre and post PR variations were calculated with a nonparametric Wilcoxon test and Spearman rank correlation test was used to assess the relationship between measures.

Abstract P146 Table 1 Response to pulmonary rehabilitation

\begin{tabular}{lccccc}
\hline Outcome measures & Before PR & After PR & Change & SD & p Value \\
\hline CAT & 20.5 & 17.3 & -3.2 & 5.5 & $<0.01$ \\
Number climbed stairs & 45.1 & 162.1 & 113.1 & 63.0 & $<0.01$ \\
Waist circumference (cm) & 100.1 & 99.6 & -1.5 & 1.5 & $<0.01$ \\
Mean bicycle power (watts) & 28.6 & 37.6 & 9.0 & 4.9 & $<0.01$ \\
Walking distance (km) & 0.72 & 0.89 & 0.17 & 0.07 & $<0.01$ \\
Bicycling distance (km) & 3.77 & 4.54 & 0.77 & 0.41 & $<0.01$ \\
Hand gripping strength (lbs) & 76.4 & 80.1 & 4.4 & 8.0 & $<0.01$ \\
\hline
\end{tabular}

Results The CAT decreased significantly by a mean of $3.2(p<0.01)$ following PR. The mean CAT score change was similar in all pulmonary disease groups and between obstructive and non obstructive diseases. The pre and post CAT score negative variation was $4.16(p<0.05)$ for women, $3.74(p<0.05)$ for patients younger than 70 years old and $4.41(p<0.05)$ for patients with $<1$ exacerbation yearly. No correlation between the CAT score change and the $\mathrm{FEV}_{1}(\mathrm{r}=-0.11)$ was found. Of all the outcome measures, the number of climb stairs was the most responsive with an amelioration of $250 \%$ after $\mathrm{PR}$.

Conclusion Even if the CAT was initially developed for the assessment of COPD patients, our study has shown that the 